\title{
Reacciones adversas por antibióticos en una unidad de cuidado intensivo pediátrico y neonatal de Bogotá
}

\author{
Alvaro Vallejos \\ Departamento de Farmacia, Universidad Nacional de Colombia, Bogotá D.C., Colombia.
}

Introducción. Los pacientes críticos tienen alta probabilidad de presentar reacciones adversas a los medicamentos por la politerapia farmacológica que reciben, pues incluye esquemas con dos o más antibióticos. La mayoría de antibióticos tienen alto riesgo de toxicidad, que puede potencializarse al asociarse con otros medicamentos.

Objetivo. Detectar y describir las reacciones adversas por antibióticos en pacientes de cuidado intensivo pediátrico y neonatal.

Materiales y métodos. Se realizó un estudio observacional descriptivo de seguimiento de una cohorte sin grupo de control con seguimiento diario durante tres meses de los pacientes. Se evaluó causalidad con la escala de Naranjo.

Resultados. Se incluyeron 85 pacientes en el estudio, 36,5\% en cuidado intensivo neonatal y $63,5 \%$ en pediátrico. Se administraron 27 antibióticos y se detectaron 97 reacciones adversas para 15 de estos en 37 pacientes (20 neonatos y 17 niños mayores). Se clasificaron como leves el $65 \%$, moderadas, $35 \%$ y ninguna como grave. Según la escala de Naranjo, $68 \%$ fueron posibles, $32 \%$, probables y no hubo definidas. Por exámenes paraclínicos se detectó nefrotoxicidad en $38,1 \%$, hematotoxicidad en $24,7 \%$, alteraciones electrolíticas en $21,6 \%$ y hepatotoxicidad en $15,5 \%$. La distribución de reacciones adversas fue de 20,6\% para gentamicina, $17,5 \%$ para vancomicina, $16,5 \%$ para amikacina, $15,5 \%$ para ceftriaxona y $13,4 \%$ para piperacilina tazobactam.

Conclusiones. La proporción de reacciones adversas fue de 43,7\%. El 55,6\% de los antibióticos ocasionó reacciones adversas. Es necesario un programa de farmacovigilancia institucional coordinado por médicos fármacólogos o químicos farmacéuticos.

Palabras clave: farmacoepidemiología, agentes antibacterianos/efectos adversos, hospitalización, unidades de cuidado intensivo pediátrico.

\section{Adverse reactions by antibiotics in a pediatric and neonatal intensive care unit located in Bogotá, Colombia}

Introduction. Critical care patients have a high probability of presenting adverse drugs reactions because the polytherapy that they receive usually include schemes with two or more antibiotics. Most antibiotics have high toxicity risk, which can be potentialized when being associated with other drugs.

Objective. Adverse reactions were detected and described in patients treated with antibiotics in a pediatric and neonatal intensive care.

Materials and methods. A follow up, descriptive and observational study of a cohort was made without control group. A daily follow-up was administered for a three month period for each patient. Causality was evaluated on the basis of the Naranjo scale.

Results. Eighty-five patients were included in the study, $36.5 \%$ from the neonatal intensive care unit and $63.5 \%$ from the pediatric clinic. Of twenty-seven antimicrobial medications administered, 97 adverse reactions were detected for 15 of these, in 37 patients (20 newborn and 17 older children). They were classified as mild (65\%), moderate (35\%) and serious (0). According to scale of Naranjo, $68 \%$ were possible, $32 \%$ probable, and defined, none. Based on laboratory test results, renal toxicity was detected in $38.1 \%$, hematotoxicity in $24.7 \%$,electrolytic abnormalities in $21.6 \%$ and hepatotoxicity in $15.5 \%$. The distribution of adverse 
antibiotic reactions was as follows: gentamycine $20.6 \%$, vancomycin $17.5 \%$, amikacine $16.5 \%$, ceftriaxone $15.5 \%$ and piperaciline tazobactam $13.4 \%$.

Conclusions. The proportion of adverse reactions was of $43.7 \% .55 .6 \%$ of antibiotics produced adverse reactions. A program of institutional pharmacovigilance coordinated by a medical pharmacologist or pharmaceutical chemist was recommended by these data.

Key words: pharmacoepidemiology, anti-bacterial agents/ adverse effects, hospitalization, intensive care units, pediatric.

Los antibióticos son medicamentos frecuentemente utilizados en el manejo de los pacientes hospitalizados, tanto para las infecciones adquiridas en la comunidad como para las nosocomiales. Su utilización no siempre es la más adecuada, más aun cuando se trata de pacientes críticos, en quienes es necesaria la utilización de esquemas múltiples con el fin de garantizar eficacia en el control de infecciones graves $(1,2)$.

Los antibióticos constituyen el segundo grupo de fármacos más utilizados en el mundo, superado sólo por los agentes cardiovasculares. En los pacientes hospitalizados existe una tendencia al incremento de los tratamientos antibióticos, los cuales se aplican aproximadamente a un 35,4\% de los pacientes, representando entre $25 \%$ y $50 \%$ del gasto farmacéutico hospitalario $(3,4)$.

Los pacientes críticos en las unidades de cuidado intensivo tienen una alta posibilidad de presentar reacciones adversas a los medicamentos (RAM), debido a que reciben terapias farmacológicas múltiples y entre ellas casi siempre están incluidos esquemas con dos o más antibióticos. La mayoría de los antibióticos tienen documentado un alto riesgo de toxicidad que frecuentemente es subvalorado y que puede potencializarse cuando se asocian a otros medicamentos $(4,5)$. Es por esto que la farmacovigilancia desempeña un papel fundamental en la prevención y detección oportuna de las reacciones adversas en este tipo de pacientes $(1,6)$.

Diversos estudios indican que las reacciones adversas a los medicamentos pueden ocurrir aproximadamente en $10 \%$ a $20 \%$ de los pacientes

\footnotetext{
$\overline{\text { Correspondencia: }}$

Álvaro Vallejos Narváez, carrera 22 No. 49-38, interior 2, apartamento 502. Teléfono: 3384881, fax: 4129942. agvallejos@gmail.com

Recibido: 21/07/06; aceptado: 11712/06
}

hospitalizados (7-9). Se ha estimado que 3\% a $7 \%$ de las admisiones a los hospitales son resultado de tales reacciones $(8,9)$, y su manejo genera un costo anual de 3 billones de dólares (7). Adicionalmente, $10 \%$ a $20 \%$ de los pacientes hospitalizados reportan reacciones adversas menores a medicamentos durante su estancia hospitalaria $(7,8)$. El porcentaje de notificación de reacciones adversas a medicamentos durante las hospitalizaciones puede variar de $1,5 \%$ a $43,5 \%(7,9)$.

Aproximadamente entre $30 \%$ y $80 \%$ de las reacciones adversas a medicamentos son prevenibles y el paso esencial en la maduración de un programa de farmacovigilancia debe ser la prevención de los problemas relacionados con medicamentos (10).

El panorama de la farmacovigilancia en Colombia es promisorio merced a los continuos esfuerzos de las instituciones hospitalarias y del Instituto Nacional de Vigilancia de Medicamentos y Alimentos (INVIMA), ente encargado de canalizar los reportes a nivel nacional, para formar una red que vigile el comportamiento de los medicamentos en la población colombiana (11).

El objetivo de este estudio fue detectar las reacciones adversas ocasionadas por los antibióticos administrados a los pacientes de la unidad de cuidado intensivo (UCI) pediátrica y neonatal de un hospital en Bogotá, describir los factores de riesgo de los pacientes probablemente asociados con la presentación de reacciones adversas ocasionadas por los antibióticos y documentar al personal asistencial de la $\mathrm{UCl}$ sobre la importancia, forma de detección y prevención de reacciones adversas a medicamentos. Se escogieron para la realización del estudio las unidades de cuidado intensivo pediátrico y neonatal debido a la alta frecuencia de utilización de antibióticos detectada por el 
seguimiento de la oficina de vigilancia epidemiológica institucional en estos dos servicios, lo que no sucede en los otros servicios del hospital. La elección también se explica porque no hay descripción amplia en la literatura de estudios orientados a la detección específica de reacciones adversas por antibióticos, a pesar de que se utilizan con mucha frecuencia a nivel intrahospitalario.

\section{Materiales y métodos}

Se realizó un estudio observacional descriptivo de seguimiento de una cohorte sin grupo de control. El estudio se llevó a cabo en un hospital pediátrico universitario de tercer nivel de atención ubicado en Bogotá D.C., Colombia, donde la unidad de cuidado intensivo pediátrico cuenta con un total de 14 camas y la neonatal con 16 camas. El protocolo de investigación fue sometido a consideración del Comité de Ética e Investigación de la institución, el cual dio la aprobación respectiva para la realización del estudio.

\section{Población de estudio}

Se incluyeron los pacientes hospitalizados en la unidad de cuidado intensivo pediátrica y neonatal en el período comprendido entre el 1 de septiembre y el 30 de noviembre de 2005 que recibieron antibióticos durante su tratamiento hospitalario y a quienes se les hizo seguimiento durante el tiempo de hospitalización. Los pacientes debían tener los exámenes paraclínicos básicos para la evaluación de reacciones adversas, tales como cuadro hemático, parcial de orina, pruebas de función renal (nitrógeno ureico, creatinina, depuración de creatinina, entre otros), pruebas de función hepática (transaminasas, fosfatasa alcalina, bilirrubinas, entre otros), y electrolitos séricos entre los más frecuentes. No se determinaron específicamente criterios de exclusión debido a que la presencia de alguna alteración a nivel hepático, renal, hematológico, enzimático o electrolítico eleva la susceptibilidad frente a reacciones adversas a los antibióticos. Además, cuando un paciente ingresa a la unidad de cuidado intensivo es muy probable que ya haya recibido tratamiento farmacológico previo y pueda estar generando signos de toxicidad, siendo muy difícil precisar si estas alteraciones al ingreso a $\mathrm{UCI}$ se tratan o no de una reacción adversa a los medicamentos recibidos previamente.

\section{Detección de los casos}

Se capacitó a tres estudiantes de medicina y al enfermero profesional de la oficina de vigilancia epidemiológica de la institución para tener su apoyo en la etapa de recolección de la información, la cual siempre fue supervisada y verificada por el investigador. Se llevó a cabo una prueba piloto de recolección de información.

La información se recolectó durante tres meses. Se utilizó una estrategia activa de seguimiento diario de la evolución clínica y de los exámenes paraclínicos de los pacientes para evaluar si había o no signos sugestivos de reacción adversa a medicamentos durante todo el tiempo de hospitalización del paciente.

\section{Definición de los casos}

Se aplicó la definición de reacciones adversas a medicamentos establecida por la Organización Mundial de la Salud (OMS) (1972) (12). Se aplicó la escala de Naranjo y colaboradores para la evaluación de causalidad de las reacciones adversas a medicamentos, clasificándolas como posible ( 1 a 4 puntos), probable (5 a 8 puntos) y definitiva (9 o + puntos) (13). La calificación de la causalidad se hizo por consenso con los otros integrantes del equipo de recolección de la información, así como con el criterio de los médicos tratantes. Se tuvieron en cuenta los signos clínicos, pero principalmente los hallazgos de los exámenes paraclínicos, ya que por tratarse de pacientes críticos y pediátricos, los signos clínicos no eran fácilmente detectables. Los casos detectados se revisaron con los médicos tratantes para promover las medidas de intervención pertinentes.

\section{Análisis de datos}

La gravedad de las reacciones adversas a medicamentos se clasificó con base en la tabla de la OMS como leve, moderada y grave (12), y teniendo en cuenta las consideraciones del médico según las circunstancias particulares del paciente. La evolución se analizó según mejoría, secuelas o fallecimiento por la reacción adversa. 
Se construyó una base de datos en Excel Microsoft Office 2003, en la cual se realizó una parte del análisis estadístico con el apoyo de Epiinfo versión 2000 cuando fue necesario. Se efectuó fundamentalmente un análisis de tipo descriptivo. Para las variables cualitativas se calcularon proporciones y para las cuantitativas, medidas de resumen, medidas de posición y dispersión, entre otras. Se utilizaron intervalos de confianza del $95 \%$ para la estimación de parámetros de verificación de la precisión de los datos estadísticos.

\section{Resultados}

\section{Generalidades}

Durante el período de estudio fueron hospitalizados en la UCI 120 pacientes; se realizó seguimiento a 85 niños porque recibieron tratamiento con antibióticos, antifúngicos y antivirales. Del total de los pacientes del estudio, el 36,5\% (31 pacientes) ingresaron en la $\mathrm{UCl}$ neonatal y el $63,5 \%$ (54 pacientes), en la pediátrica. El 60\% (51 pacientes) era de género masculino y el $40 \%$ (34 pacientes), de género femenino.

En la UCI neonatal, el promedio de edad fue de $18,8 \pm 9$ días con un rango de 1 a 41 días. En la $\mathrm{UCI}$ pediátrica, la edad promedio fue de $43 \pm 51$ meses con un rango de 2 a 207 meses (17,3 años). El peso promedio de los neonatos fue de $3 \pm 0,7$ kg (IC 95\%: 3,8 a 3,2 kg) y en los niños de la UCl pediátrica estuvo en $14,2 \pm 12 \mathrm{~kg}$ (IC 95\%:11 a $17,4 \mathrm{~kg}$ ).

\section{Hallazgos clínicos}

Al evaluar los antecedentes personales que podían relacionarse en algún momento con riesgo para la presentación de reacción adversa a medicamentos (5), se encontraron antecedentes alérgicos en el 5,9\% (cinco pacientes) y antecedente de alteraciones enzimáticas en el $1,2 \%$ (un paciente). En cuanto a los antecedentes familiares que en algún momento podían relacionarse con riesgo para la presentación de las reacciones (5), solamente en el 2,4\% (dos pacientes) se refirieron antecedentes, en uno alteraciones enzimáticas y en otro alteraciones hepáticas.
Las patologías infecciosas por las cuales los pacientes recibieron tratamiento con antibióticos se pueden observar en el cuadro 1 . Con relación a las patologías diferentes a las infecciosas y que podían relacionarse con riesgo para la presentación de reacciones adversas a medicamentos, el 54\% (IC 95\%:43 a 64\%) (46 pacientes) presentó alguna de las siguientes: ictericia neonatal, 11,8\% (10); prematurez, 9,4\% (8); deshidratación, 5,9\% (5); cardiopatía congénita, 4,7\% (4); síndrome anémico, 3,5\% (3), y diabetes mellitus, 2,4\% (2).

Para la clasificación del nivel de estado clínico crítico de los pacientes hospitalizados en la UCI pediátrica se utilizó la escala de TISS (Sistema de puntuación de intervenciones terapéuticas en pacientes críticos), la cual fue aplicada por los médicos pediatras intensivistas. Según las guías de manejo clínico institucional, dicha escala solamente se aplica a los pacientes de UCI pediátrica. Se aplicó a 54 pacientes que fueron clasificados como TISS I (menor estado crítico: $74,1 \%, 40$ pacientes), TISS II (20,4\%, 11 pacientes) y TISS III (5,6\%, 3 pacientes); ningún paciente fue calificado como TISS IV (mayor estado crítico).

Cuadro 1. Causas de morbilidad infecciosa en pacientes internados en UCI pediátrica y neonatal de un hospital de tercer nivel de atención de Bogotá, D.C.

\begin{tabular}{lcc}
\hline Diagnóstico infeccioso & Frecuencia & Porcentaje \\
\hline Sepsis neonatal & 18 & 21,2 \\
Bronconeumonía & 9 & 10,6 \\
Conjuntivitis bacteriana & 9 & 10,6 \\
Infección urinaria adquirida & & \\
en la comunidad & 8 & 9,4 \\
Neumonía adquirida en la & & \\
comunidad & 6 & 7,1 \\
Enterocolitis & 5 & 5,9 \\
Infección urinaria nosocomial & 5 & 5,9 \\
Bronquiolitis sobreinfectada & 4 & 4,7 \\
Síndrome broncobstructivo & & \\
sobreinfectado & 4 & 4,7 \\
Otitis media aguda & 4 & 4,7 \\
Sepsis & 4 & 4,7 \\
Laringotraqueobronquitis & 3 & 3,5 \\
Neumonía nosocomial & 3 & 3,5 \\
Toxoplasmosis congénita & 3 & 3,5 \\
Celulitis & 2 & 2,4 \\
Total pacientes & 85 & \\
\hline UCl: Unidad de cuidado intensvo & & \\
\hline
\end{tabular}

UCl: Unidad de cuidado intensivo 
La mediana de los días de estancia hospitalaria de los pacientes del estudio fue de 12, con una moda de seis días, un valor mínimo de tres días, un máximo de 75 días de hospitalización y un rango de 72 días.

\section{Descripción general de los antibióticos y otros medicamentos administrados}

Respecto a los días de seguimiento del tratamiento con antibióticos en los pacientes, la mediana fue de nueve días, con una moda de seis días, un valor mínimo de tres días, un valor máximo de 44 días y un rango de 41 días. La mediana del número de antibióticos que recibieron los pacientes durante su estancia hospitalaria fue de dos, con una moda igualmente de dos y un rango de 7 (R: 1 a 8). Los otros medicamentos diferentes a antibióticos administrados a los pacientes tuvieron una mediana de cinco, una moda de dos y un rango de 10 (R: 1 a 11). Para el total de medicamentos recibidos por los pacientes (antibióticos + otros medicamentos), la mediana fue de cinco, con una moda de dos y un rango de 10 (R: 1 a 11).

Se formularon en la UCI pediátrica y neonatal un total de 27 antibióticos; la frecuencia de utilización se puede observar en el cuadro 2. Al analizar los tipos de antibióticos, los más formulados fueron los de la familia de las penicilinas en $77,6 \%$ (66 pacientes) (IC 95\%: 67\% a 88\%), aminoglicósidos en $57,6 \%$ (49 pacientes) (IC $95 \%$ : $47 \%$ a $68 \%$ ), cefalosporinas en $36,5 \%$ (31 pacientes) (IC 95\%: $26 \%$ a $47 \%$ ) e inhibidores de las betalactamasas (se incluyeron aquí ampicilina sulbactam y piperacilina tazobactam) en $23,5 \%$ (20 pacientes) (IC 95\%: 13\% a 34\%). Los antibióticos de la familia de los glicopéptidos (particularmente vancomicina) se formularon en $12,9 \%$ (11 pacientes) y los carbapenems sólo en 4,7\% (cuatro pacientes).

En la UCI neonatal, la ampicilina se utilizó en $77,4 \%$ de los pacientes que requirió tratamiento con antibióticos y gentamicina en $87,1 \%$. Otros antibióticos se utilizaron según los protocolos institucionales para el manejo de las patologías infecciosas solamente en aquellos casos en los cuales no había respuesta a los dos antibióticos mencionados, teniendo en cuenta el criterio de uso escalonado y racional. En la $\mathrm{UCI}$ pediátrica, la utilización de antibióticos fue más diversa; la
Cuadro 2. Antibióticos formulados a los pacientes internados en las UCI pediátrica y neonatal de un hospital de Bogotá.

\begin{tabular}{lcc}
\hline Antibiótico & $\begin{array}{c}\text { No. de veces } \\
\text { formulado }\end{array}$ & Porcentaje \\
\hline Ampicilina & 36 & 42,4 \\
Gentamicina & 31 & 36,5 \\
Amikacina & 17 & 20,0 \\
Cefazolina & 13 & 15,3 \\
Ceftriaxona & 13 & 15,3 \\
Ampicilina sulbactam & 12 & 14,1 \\
Oxacilina & 12 & 14,1 \\
Vancomicina & 11 & 12,9 \\
Sulfacetamida & 10 & 11,8 \\
Amoxicilina & 9 & 10,6 \\
Penicilina cristalina & 9 & 10,6 \\
Piperacilina tazobactam & 8 & 9,4 \\
Metronidazol & 6 & 7,1 \\
Clotrimazol & 4 & 4,7 \\
Clindamicina & 3 & 3,5 \\
Total pacientes & 85 & \\
\hline UCl: Unidad de cuidado & &
\end{tabular}

UCI: Unidad de cuidado intensivo

amikacina se utilizó en 18,5\% de los pacientes, la ceftriaxona en $24,1 \%$ y la oxacilina en $16,7 \%$.

El 100\% (IC 95\%: $89 \%$ a $110 \%$ ), o sea los 85 pacientes, recibió los antibióticos por vía intravenosa y $21,1 \%$ (18 pacientes), por otras vías como la oral. El único medicamento utilizado por vía tópica fue el clotrimazol en $4,8 \%$ (cuatro pacientes); por vía ocular se administraron en 11 pacientes (12,9\%).

\section{Hallazgos generales sobre reacciones adversas a medicamentos}

Se hallaron 97 reportes de reacciones adversas a medicamentos en 37 pacientes (43,7\%) (IC 95\%: $33 \%$ a $54 \%$ ) del total de 85 pacientes del estudio. La proporción de reacciones detectadas en la $\mathrm{UCI}$ neonatal fue de $64,5 \%$ (IC 95\%: 47\% a 82\%) (20 de 31 pacientes) y en los pacientes de la UCI pediátrica fue de $31,5 \%$ (IC 95\%: 18\% a 45\%) (17 de 54 pacientes). La clasificación según la gravedad fue la siguiente: leve, 64,9\% (IC 95\%: $56 \%$ a $77 \%$ ) (63 casos); moderada, 35,1\% (IC 95\%: $23 \%$ a $45 \%$ ) (34 casos), y ningun paciente fue calificado como grave.

Al evaluar la causalidad de las reacciones adversas a medicamentos con la escala de Naranjo, el 68\% (IC 95\%: 57\% a $78 \%$ ) fue catalogado como posible (1 a 4 puntos) y el $32 \%$ 
(IC 95\%: $23 \%$ a $43 \%$ ) como probable (5 a 8 puntos). Ninguna de estas reacciones alcanzó la calificación de definitiva (9 o más puntos). En 20 $(54,5 \%)$ de los 37 pacientes que presentaron reacciones adversas a medicamentos, los médicos tratantes aceptaron que podían ser secundarios a los medicamentos y tomaron medidas de intervención tales como disminución de la dosis en 19 pacientes $(51,4 \%)$; solamente en un paciente $(2,7 \%)$ se cambió el medicamento. Los médicos tratantes consideraron que en algunos pacientes, los hallazgos sugestivos se trataban de situaciones clínicas esperadas en la patología de base.

Se encontraron reacciones relacionadas con signos de nefrotoxicidad (aumento de creatinina sérica o nitrógeno uréico) en 38,1\% (IC 95\%: 28\% a 48\%) (37 casos), alteraciones hema-tológicas (anemia, leucocitopenia, trombocitopenia, trombocitosis) en $24,7 \%$ (IC 95\%: $15 \%$ a $35 \%$ ) (24 casos), alteraciones electrolíticas (aumento o disminución de sodio, potasio, cloro, magnesio, calcio y fosfato) en $21,6 \%$ (IC 95\%: $12 \%$ a $31 \%$ ) ( 21 casos) y reacciones relacionadas con signos de hepatotoxicidad (aumento de transaminasas o bilirrubinas y fosfatasa alcalina) en 15,5\% (IC 95\%: $6 \%$ a $25 \%$ ) (15 casos). No se detectaron casos de reacciones dermatológicas, digestivas ni neurológicas.

Los signos clínicos de reacciones adversas a medicamentos fueron escasos; se encontraron signos de flebitis de tipo químico en seis pacientes en el sitio de venopunción, ictericia en cuatro, hematuria en tres, y en otros dos, coagulopatías manifestadas por equimosis pequeñas.

\section{Hallazgos específicos de reacciones adversas a medicamentos según antibióticos}

No se identificaron reacciones para 12 de los antibióticos (44,4\%): aciclovir, amoxicilina, cefalexina, cefepima, ciprofloxacina, claritromicina, clindamicina, clotrimazol, eritromicina, metronidazol, sulfacetamida y tobramicina. Con $55,6 \%$ (IC 95\%: 37 a 54\%) (15 antibióticos) se encontraron reacciones adversas.

Del total de reacciones identificadas, $20,6 \%$ correspondió a gentamicina (IC 95\%: 11 a 30\%) (20 casos), seguida en orden descendente por
17,5\% con vancomicina (IC 95\%: $8 \%$ a $27 \%$ ) (17 casos), 16,5\% con amikacina (IC 95\%: 7\% a 26\%) (16 casos), $15,5 \%$ con ceftriaxona (IC $95 \%$ : $6 \%$ a $25 \%$ ) (15 casos) y $13,4 \%$ con piperacilinatazobactam (IC 95\%: 4\% a 23\%) (13 casos).

Los antibióticos más frecuentemente utilizados fueron piperacilina tazobactam, vancomicina, ceftriaxona, amikacina, gentamicina, ampicilina y oxacilina; el cuadro 3 muestra la proporción de reacciones adversas con base en la frecuencia de utilización de cada antibiótico y el cuadro 4 muestra detalladamente los hallazgos de reacciones que se presentaron según el sistema afectado y el antibiótico específico.

\section{Reacciones adversas por medicamentos diferentes a antibióticos}

Se detectaron cinco medicamentos diferentes a antibióticos que probablemente tuvieron alguna relación con la aparición de reacciones adversas; estos fueron detectados en 55 casos y correspondieron principalmente a medicamentos como furosemida en $47,3 \%$ (IC 95\%: 34\% a 60\%) (26 casos), corticoides (prednisolona, metilprednisolona, flucortisona, hidrocortisona) en $25 \%$ (IC $95 \%$ : $12 \%$ a $38 \%$ ) (14 casos), captopril en $25 \%$

Cuadro 3. Proporción de reacciones adversas para algunos antibióticos en pacientes de UCl pediátrica y neonatal de un hospital de Bogotá.

\begin{tabular}{lrcr}
\hline $\begin{array}{l}\text { Antibiótico asociado } \\
\text { con reacción adversa }\end{array}$ & $\begin{array}{c}\text { No. } \\
\text { RAM }\end{array}$ & $\begin{array}{c}\text { No. veces } \\
\text { utilizado }\end{array}$ & $\begin{array}{c}\text { Porcentaje } \\
\text { RAM }\end{array}$ \\
\hline Meropenem & 2 & 2 & 100,0 \\
Anfotericina B & 1 & 1 & 100,0 \\
Cefotaxima & 1 & 1 & 100,0 \\
Piperazilina tazobactam & 7 & 8 & 87,5 \\
Vancomicina & 7 & 11 & 63,6 \\
Ceftriaxona & 8 & 13 & 61,5 \\
Amikacina & 10 & 17 & 58,8 \\
Imipenem & 1 & 2 & 50,0 \\
Pirimetamina & 1 & 2 & 50,0 \\
Gentamicina & 12 & 31 & 41,4 \\
Ampicilina & 13 & 36 & 36,1 \\
Oxacilina & 4 & 12 & 33,3 \\
Ampicilina Sulbactam & 2 & 12 & 16,1 \\
Penicilina Cristalina & 1 & 9 & 11,0 \\
Cefazolina & 1 & 13 & 9,7 \\
Total & 71 & & \\
\hline
\end{tabular}

RAM: Reacción adversa a medicamento

UCI: Unidad de cuidado intensivo 
Cuadro 4. Tipo de reacciones adversas según antibiótico en pacientes de las UCI pediátrica y neonatal de un hospital de Bogotá.

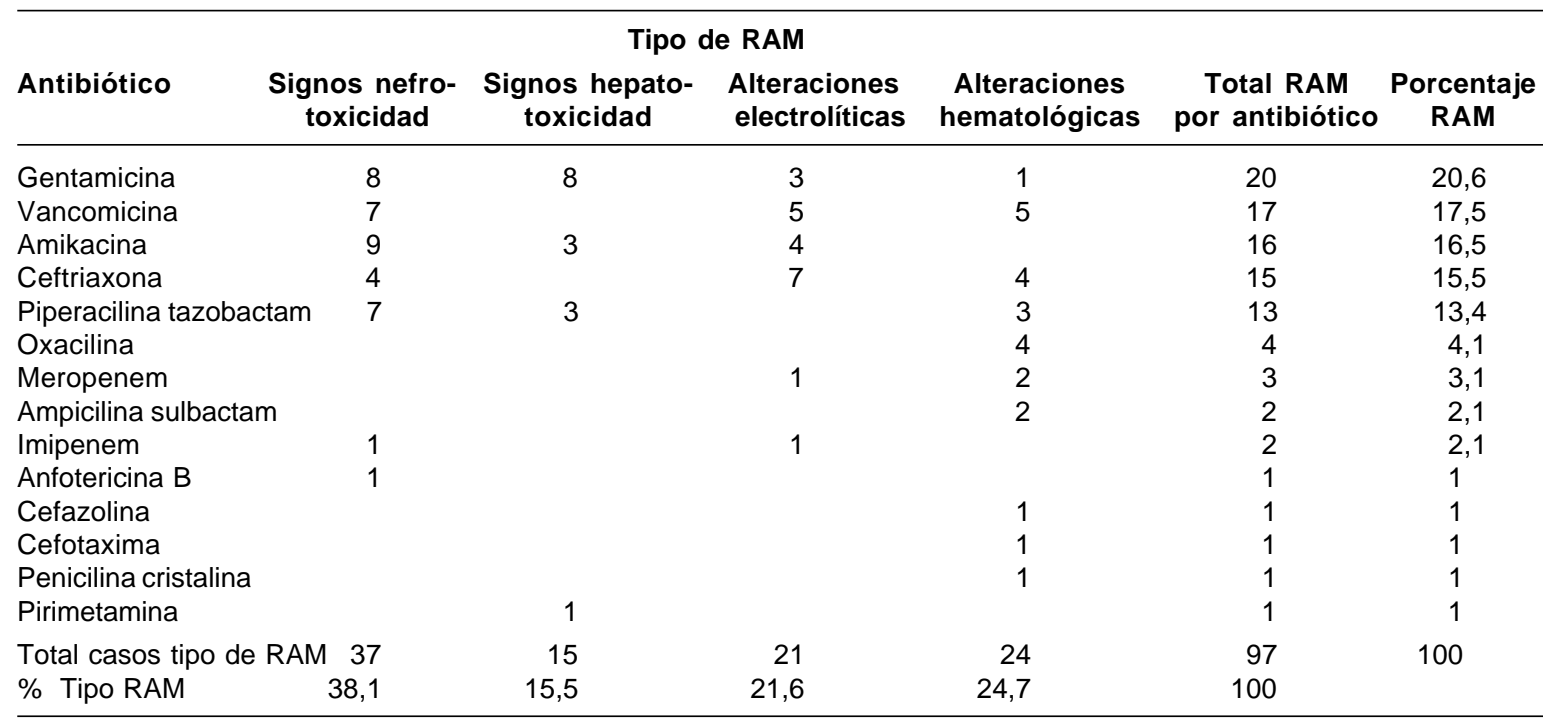

RAM: Reacción adversa a medicamento

UCl: Unidad de cuidado intensivo

(IC 95\%: 12\% a 38\%) (14 casos) e indometacina en $1,8 \%$ (un caso).

Se encontraron reacciones relacionadas directamente con medicamentos diferentes a los antibióticos con tres de ellos: metilprednisolona (tres casos), prednisolona (dos casos) y furosemida (un caso). En los seis casos se encontraron signos relacionados con alteraciones electrolíticas así: hipercalemia en dos casos $(33,3 \%)$, hipermagnesemia en los seis pacientes $(100 \%)$ e hipofosfatemia en dos pacientes $(33,3 \%)$. En total se hallaron 10 reacciones adversas para estos medicamentos.

\section{Desenlace de las reacciones adversas a medicamentos}

La evolución del total de reacciones adversas a medicamentos fue hacia la mejoría. La proporción de complicaciones serias que pudiera relacionarse con ellas fue de cero.

\section{Discusión}

La detección de reacciones adversas a medicamentos se realizó principalmente a través de los exámenes paraclínicos; en muy pocos casos se evidenciaron hallazgos clínicos, debido al estado crítico de los pacientes y a su corta edad, lo cual dificulta la manifestación de sintomatología alguna, y queda limitado a la detección de signos por parte de los médicos tratantes. Esto es válido para efectos adversos como los gastrointestinales (distensión abdominal, náuseas, dispepsia), mareos, cefalea, entre otros.

La frecuencia de detección en este estudio fue de 43,7\% (IC 95\%: 33\% a 54\%) (37 pacientes), cifra mayor a la de otros estudios (25,1\%) (14). Este hallazgo puede estar relacionado con el hecho de que el estudio se realizó en pacientes críticos que presentaban múltiples patologías y complicaciones. Igualmente, la inmadurez de los sistemas fisiológicos en recién nacidos altera la farmacocinética y farmacodinamia de varios medicamentos, entre ellos los antibióticos, lo cual los hace más susceptibles de complicaciones. Con base en lo descrito se puede explicar de alguna manera la mayor proporción de reacciones adversas en la UCI neonatal $(64,5 \%)$ que en la pediátrica (31,5\%); estas consideraciones se tienen en cuenta también en otros estudios $(14,15)$, en uno de los cuales se encontraron 268 reacciones en 208 pacientes (14); en el presente estudio fueron 97 en 37 pacientes. 
Al efectuar la clasificación según gravedad, se encontró que el $65 \%$ (IC 95\%: $56 \%$ a $77 \%$ ) fue leve; el 35\% (IC 95\%: 23\% a 45\%) moderada, y no se encontró ninguna grave; estos hallazgos difieren de los otros estudios (14), en los que los porcentajes fueron del $41 \%$ y $53,7 \%$ para las leves y moderadas, respectivamente. Hubo una diferencia clara en el hallazgo de reacciones graves, ya que en este estudio se clasificó así el $7,6 \%$ de las reacciones. En el presente estudio se clasificó como grave la hipercalemia como signo de reacción adversa a gentamicina, amicacina, ceftriaxona, vancomicina y piperacilina tazobactam, aunque se presentó en pocos pacientes y con niveles de potasio ligeramente por encima de $5,5 \mathrm{mEq} / \mathrm{L}$, ya que en algún momento puede generar riesgo de arritmias cardíacas. Otras publicaciones $(14,16)$ mencionan unos valores similares en cuanto a la distribución de la gravedad de las reacciones.

La evaluación de la causalidad con la escala de Naranjo mostró diferencias importantes con otros estudios. En éste, la clasificación de posibles fue para el $68 \%$ y en otros solamente del $11,1 \%$ (14); lo mismo aplica para la clasificación de probables con $32 \%$ y $74,2 \%$ (14) y para la de definitivas, que en nuestra caso fue de $0 \%$ y en otro estudio fue de $11,1 \%$ (14). Este hecho puede relacionarse con la circunstancia de que las personas evaluadas en el otro estudio (14) eran pacientes del servicio de medicina interna, en quienes las reacciones pueden ser mucho más frecuentes, por ser en su gran mayoría de edad avanzada, que en los pacientes pediátricos, que tienen mejor capacidad para manejar los medicamentos y se recuperan más rápidamente, con excepciones en los neonatos para ciertos medicamentos. En otros estudios (17) se han obtenido hallazgos similares (58,7\%, posibles, $29,5 \%$, probables, pero $11,8 \%$, definidas). Es de anotar que los métodos utilizados para la determinación de causalidad de las reacciones muestran mucha variación en y entre evaluadores y no han podido ser validados completamente.

La alta proporción de reacciones adversas con piperacilina-tazobactam, vancomicina, ceftriaxona y amikacina es similar a la encontrada en otro estudio (18), el cual arrojó, por ejemplo, un porcentaje de $63,6 \%$ (IC $95 \%$ : $45 \%$ a $93 \%$ ) con la vancomicina. Las reacciones adversas con estos antibióticos se expresaron principalmente a través de signos de nefrotoxicidad, los cuales pueden desembocar en trastornos electrolíticos, así como de alteraciones hematológicas y hepáticas (5).

El estudio evidenció que las reacciones adversas pueden detectarse tempranamente si se realiza un seguimiento específico de los pacientes con tal finalidad y permite informar a los médicos tratantes de su presencia. Un estudio encontró que la participación de un químico farmacéutico dedicado al seguimiento farmacoterapéutico del paciente puede disminuir sustancialmente la posibilidad de que se presenten RAM hasta en un $66 \%$ (16). Es de resaltar la disposición de los médicos tratantes para intervenir ante la presencia de una posible reacción adversa, lo cual sucedió en $54,5 \%$ de los casos, ya que las sugerencias del investigador fueron tomadas en cuenta en 20 de los 37 pacientes que presentaron algún evento adverso. La aceptación pudo estar influída por el hecho de que el investigador era médico y laboraba en la institución. En la mayoría de los casos, la aceptación de los médicos tratantes es baja; un estudio, sin embargo, mostró diferencias importantes (16), ya que de las 366 recomendaciones presentadas por el químico farmacéutico para los pacientes de la unidad de cuidado intensivo relacionadas con irregularidades en la prescripción de los medicamentos que potencialmente pudieron haber desencadenado reacciones adversas, 362 (99\%) fueron aceptadas por los clínicos.

Sobre el desenlace de las reacciones adversas a medicamentos, un estudio mostró que el $98 \%$ se resolvió sin secuelas, el 0,7\% ocasionó secuelas y el $1,1 \%$ (tres pacientes) fallecieron (14). En el presente estudio se evidenció que el $100 \%$ de los pacientes evolucionaron hacia la mejoría sin secuelas. Sin embargo, es importante tener en cuenta que con algunos antibióticos como la vancomicina y los aminoglicósidos, las secuelas pueden darse a largo plazo, y su presencia escapa a los objetivos del presente estudio porque no fue diseñado para realizar tal seguimiento y detectar signos de ototoxicidad, por ejemplo. La 
mayoría de los signos de nefrotoxicidad con casi todos los medicamentos es de escasa duración y revierte poco tiempo después de la suspensión del medicamento (5).

En la realización de este estudio es importante aclarar que ante la sospecha de un hallazgo sugestivo de reacción adversa al medicamento, y por consenso entre las personas encargadas de recoger la información y el investigador, se definía si se trataba o no de reacción adversa apoyándose en la revisión bibliográfica sobre los antibióticos. Se efectuó un análisis conjunto con los médicos tratantes en algunos casos de duda debida a las características de la patología de base. La clasificación de gravedad y evolución de causalidad no se hizo con dos evaluadores independientes sino en consenso con los integrantes del grupo de investigación.

Los criterios de inclusión del estudio se definieron de manera muy amplia como se hace en la mayoría de los estudios de farmacovigilancia con el fin de incluir la mayor parte de los pacientes, incluso aquellos que pueden tener ya signos manifiestos de alteraciones hepáticas, renales 0 hematológicas que pueden estar relacionadas con medicamentos recibidos en otros servicios antes del ingreso a la $\mathrm{UCl}$. Es conveniente mencionar la limitación que implican las alteraciones halladas en los exámenes paraclínicos antes del uso de los antibióticos, ya que puede ser una variable de confusión.

Es importante tener en cuenta que, por tratarse de pacientes críticos, pudieron existir algunos factores capaces de generar en alguna instancia afecciones en los diferentes sistemas, haciendo más difícil la definición de si era o no una reacción adversa debida al medicamento. Cabe mencionar que los antibióticos y otros medicamentos administrados al paciente, si no desencadenan directamente estas alteraciones en los pacientes, pueden agravarlas, lo que se consideraría una reacción adversa. Debido al estado crítico de los pacientes y la cantidad de medicamentos que habitualmente reciben en la unidad de cuidado intensivo, se hace difícil establecer de manera totalmente clara el papel específico del antibiótico en las complicaciones que pueda generar y que puedan relacionarse con una reacción. Esta misma consideración se tuvo en cuenta en otro estudio (1).

La proporción de incidencia de reacciones adversas a los medicamentos en los pacientes de cuidado intensivo que recibieron antibióticos en este estudio fue del $43,7 \%$, mayor a lo reportado en otros estudios, pero cabe señalar que la frecuencia de detección de estas reacciones no depende sólo del servicio evaluado, sino también de otros factores como el método y los criterios de detección.

La prescripción de antibióticos en la UCI pediátrica y neonatal se ajustó a lo reportado en la literatura.

Los exámenes paraclínicos son de gran ayuda para la detección y seguimiento de reacciones adversas a medicamentos en pacientes pediátricos críticos, considerando que su corta edad y su estado crítico hace muy difícil, a veces casi imposible, identificarlas y es muy probable que muchas de ellas se pasen inadvertidas. En este estudio la gran mayoría fueron identificadas por los exámenes paraclínicos.

El personal asistencial en la unidad de cuidado intensivo no está sensibilizado en cuanto a la importancia de la prevención, detección oportuna y seguimiento, así como tampoco hay un conocimiento adecuado de las interacciones farmacológicas y reacciones adversas a los antibióticos.

Es fundamental que las instituciones hospitalarias estructuren un programa de farmacovigilancia coordinado por un médico farmacólogo con el apoyo de un químico farmacéutico de dedicación completa a las actividades de farmacovigilancia para así garantizar el proceso de sensibilización, capacitación, acompañamiento, detección oportuna y seguimiento de las reacciones adversas y los problemas relacionados con la administración de los medicamentos a los pacientes, el análisis de casos y la notificación de los eventos adversos relacionados con los medicamentos. Ello redundaría en una atención de mayor calidad, en la disminución del riesgo para el paciente y en la optimización de los recursos. 
El estudio no se planteó como objetivo abordar los problemas relacionados con los medicamentos y el fracaso terapéutico, lo cual hace parte del contexto de evento adverso por medicamentos, ni tampoco determinar interacciones farmacológicas. Sería conveniente diseñar estudios enfocados a la evaluación específica de estos parámetros farmacológicos.

\section{Agradecimientos}

A Carlos Maldonado (director de tesis), Jorge Díaz (codirector de tesis), Alfredo Portilla (enfermero de la oficina de vigilancia epidemiológica), a la institución hospitalaria que brindó el espacio para realizar la investigación y a los cuatro estudiantes de medicina de la Universidad del Rosario por su apoyo al proyecto.

\section{Conflicto de intereses}

El autor declara que no se presentaron conflictos de intereses de orden académico, institucional ni operacional en el momento de la realización de la investigación.

\section{Financiación}

Esta investigación fue realizada por el autor con tutoría como parte de los requisitos para optar al título de Magíster en Farmacología de la Universidad Nacional de Colombia. Como tal, la investigación no contó con financiación de fuente específica (beca o subvención); los costos de la investigación fueron asumidos por el autor en su totalidad.

\section{Referencias}

1. Richards MJ, Edwards JR, Culver DH, Gaynes RP. Nosocomial infections in pediatric intensive care units in the United Status. National Nosocomial Infections Surveillance System. Pediatrics 1999;103:51-7.

2. Marin K. Optimizing antibiotic therapy in the intensive care setting. Critical Care 2001;5:189-95.

3. Arbeláez C, Ardila E, Calderón C, Cárdenas M, Chaves M, Escobar $\boldsymbol{J}$ et al. Boletín de Farmacovigilancia. Diciembre 2004. [Consultado 2005, marzo 5]. Disponible en: http://www.invima.gov.co/version1/ farmaco-vigilancia/boletindiciembre2004.htm

4. Mandell GL, Bennet JE, Dolin R, Polk RE, Fishman NO. Principles and practice of infectious diseases:
Antimicrobial management cost and resistence. Sixth Edition. Philadelphia: Elsevier Churchill Livingstone; 2005. p.611-28.

5. Goodman A. Las bases farmacológicas de la terapéutica. Décima edición. Ciudad de México: Editorial McGraw-Hill Interamericana; 2003. p.1159 -328.

6. Comisión de Farmacovigilancia - Avanzar. Manual de farmacovigilancia. Décima edición. Bogotá; Avanzar; 2002. p.18-24.

7. Cobert B, Briron T. Pharmacovigilance from A to Z: Adverse drug event surveillance. Volumen 38. Massachussets: Blackwell Science; 2003. p.29-34.

8. Rich DS. A process for interpreting data on adverse drug events: Determining optimal target levels. Clin Ther 1998;20(Suppl. C):C59-71.

9. Hartwig SC, Siegel J, Schneider PJ. Preventability and severity assessment in reporting adverse drug reactions. Am J Hosp Pharm 1998;49:2229-32.

10. Pearson F, Moore N, Fach J. Factors associated with preventable adverse drug reactions. Am J Hos Pharm 1994;51:2268-72.

11. Amariles P. El medicamento: compendio básico para su utilización correcta. Primera edición. Medellín: Impresos LTDA; 2002. p.28-35.

12. World Health Organization. International Drug Monitoring: The Role of National Centers. Technical Report Series No. 498. Geneve: WHO; 1972. [Consultado: 2005, abril 15]. Disponible en: http:// www.who-umc.org/graphics/9277.pdf

13. Naranjo CA, Busto U. Reacciones adversas a medicamentos. En: Métodos en farmacologia clínica. Santiago: OPS; 1992. p.331.

14. Tribiño C, Maldonado C, Segura O, Díaz J. Costos directos y aspectos clínicos de las reacciones adversas a medicamentos en pacientes hospitalizados en el servicio de medicina interna de una institución de tercer nivel de Bogotá. Biomédica 2006;26:31-41.

15. Lazarou J, Pomerans BH, Corey PN. Incidence of adverse drug reactions in hospitalized patient: A metaanalysis of prospective studies. JAMA 1998;279:1200-5.

16. Leape LL, Cullen DJ, Clapp MD, Burdick E, Demonaco HJ, Erickson Jl et al. Pharmacist participation on physician rounds and adverse drug events in the intensive care unit. JAMA 1999;267:282-3.

17. Evans RS, Pestotnik SL, Classen DC, Horn SD, Bass SB, Burke JP. Preventing adverse drug events in hospitalized patients. Ann Pharmacother 1994;28:523-7.

18. Lundostrom TS, Sobel JD. Antibiotics for gram positive bacterial infection: Vancomycin, quinupristin dalfopristin, linezolid and daptomycin. Infect Dis Clin North Am 2004;18:651-68. 